\title{
Familial Hypercholesterolemia with Multiple Large Tendinous Xanthomas and Advanced Coronary Artery Atherosclerosis
}

\author{
Fumio Terasaki ${ }^{1}$, Hideaki Morita ${ }^{1}$, Mariko Harada-Shiba ${ }^{2}$, Naotaka Ohta $^{3}$, Kaoru Otsuka ${ }^{1}$, \\ Shinpei Nogi ${ }^{1}$, Masatoshi Miyamura ${ }^{1}$, Shuji Suzuki ${ }^{1}$, Takahide Ito ${ }^{1}$, Hiroaki Shimomura ${ }^{4}$, \\ Takahiro Katsumata ${ }^{5}$, Yoshihiro Miyamoto ${ }^{2}$ and Nobukazu Ishizaka ${ }^{1}$
}

\begin{abstract}
We herein report the case of a 53-year-old man with severe coronary ischemia who underwent successful coronary artery bypass surgery. Of note, he had hypercholesterolemia and presented with multiple large tendinous xanthomas and thickened Achilles tendons that had been present for more than two decades. Together with a family history of dyslipidemia, the patient was diagnosed as having familial hypercholesterolemia. Irrespective of an extensive search for possible mutations in the genes presumably involved in the patient's pathophysiology, including low-density lipoprotein receptor (LDLR), proprotein convertase subtilisin/kexin type 9 (PCSK9), autosomal recessive hypercholesterolemia (ARH) and apolipoprotein B (APOB), we were not able to identify the gene mutations responsible for the phenotype observed in the present case.
\end{abstract}

Key words: familial hypercholesterolemia, tendinous xanthomas, coronary artery disease

(Intern Med 52: 577-581, 2013)

(DOI: 10.2169/internalmedicine.52.8522)

\section{Introduction}

Familial hypercholesterolemia (FH) is characterized by increased levels of serum low-density lipoprotein (LDL) cholesterol with a high prevalence of coronary atherosclerosis. It may be inherited as an autosomal dominant trait, and the frequencies of homo- and heterozygotes are estimated to be $1 / 1 \times 10^{6}$ and $1 / 500$, respectively, in the general population. Establishing a diagnosis in these patients is important because lipid-lowering therapy not only slows the progression of atherosclerosis, but also may achieve regression of atherosclerotic vascular lesions. Genetically determining the presence of $\mathrm{FH}$ is feasible, although testing is not yet available for routine use. In a large portion of patients, the diagnosis of heterozygous FH is based on laboratory and clinical criteria $(1,2)$.

\section{Case Report}

A 33-year-old man was diagnosed as having hypercholesterolemia more than two decades ago. The lipid profile of his mother at 72 years of age was as follows: total cholesterol, $257 \mathrm{mg} / \mathrm{dL}$; LDL cholesterol, $167 \mathrm{mg} / \mathrm{dL}$; high density lipoprotein (HDL) cholesterol, $72 \mathrm{mg} / \mathrm{dL}$; and triglycerides $138 \mathrm{mg} / \mathrm{dL}$. She was not taking any lipid-lowering drugs nor did she have xanthomas. We could not obtain information regarding abnormal lipid metabolism from other family members. Since then, multiple subcutaneous nodular tumorous lesions began to develop in the present patient and grew larger on the sites of the bilateral Achilles tendons, the ulnar side of the right wrist, the dorsum of the right hand and the

\footnotetext{
${ }^{1}$ Department of Cardiology, Osaka Medical College, Japan, ${ }^{2}$ Department of Molecular Innovation in Lipidology, National Cerebral and Cardiovascular Center Research Institute, Japan, ${ }^{3}$ Laboratory of Molecular Genetics, National Cerebral and Cardiovascular Center Research Institute, Japan, ${ }^{4}$ Department of Internal Medicine, Arisawa General Hospital, Japan and ${ }^{5}$ Department of Cardiovascular Surgery, Osaka Medical College, Japan

Received for publication July 4, 2012; Accepted for publication November 27, 2012
}

Correspondence to Dr. Fumio Terasaki, in3012@poh.osaka-med.ac.jp 

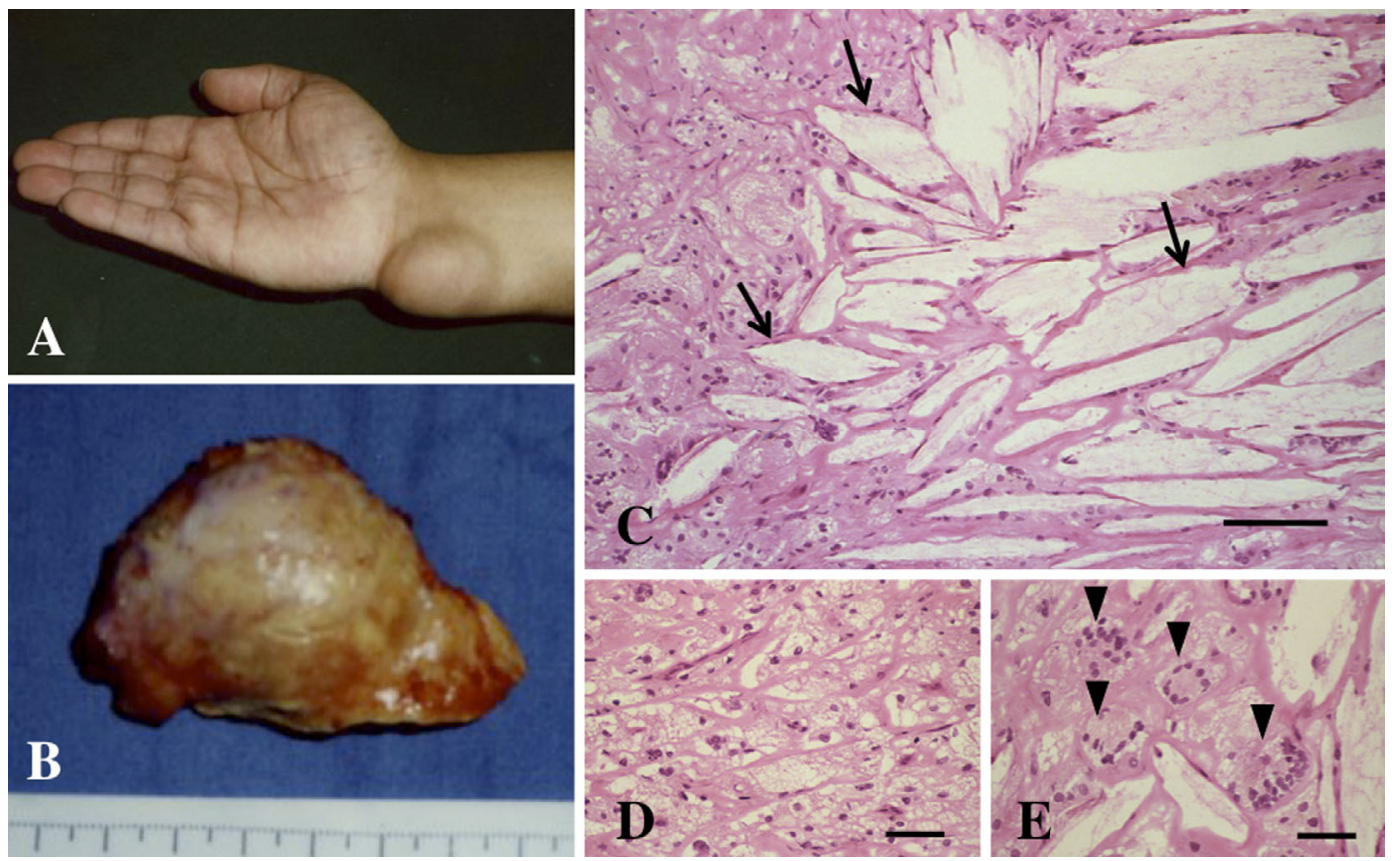

Figure 1. A nodular tumor on the ulnar side of the right wrist observed in 1999 (A). The resected tumor measured $6.0 \mathrm{~cm} \times 3.5 \mathrm{~cm}$ in size and was yellowish and lustrous (B). A microscopic examination of the resected tumor revealed numerous extracellular cholesterol deposits (cholesterol clefts) (arrows in C) and diffuse sheets of foamy cells (xanthoma cells) (D) within fibrous tissue. Multinucleated giant cells were also conspicuous (arrowheads in E). The scale bar indicates $100 \mu \mathrm{m}$ in $\mathrm{C}$ (magnification: $\times 40)$ and $50 \mu \mathrm{m}$ in D, E (magnification: $\times 100$ ).

left fingers. These subcutaneous tumors grew up to the size of a ping-pong ball or an egg. In 1999, at 41 years of age, the patient was admitted to the orthopedics department where some of the subcutaneous tumorous lesions were surgically resected (Fig. 1A, B). A histological examination of the resected specimens revealed numerous extracellular cholesterol (cholesterol clefts) and diffuse sheets of foamy cells (xanthoma cells) interspersed with inflammatory cells within fibrous tissue. Multinucleated giant cells were also conspicuous (Fig. 1C-E). A diagnosis of tendinous xanthomas was then made. The patient's serum LDL cholesterol level was $193 \mathrm{mg} / \mathrm{dL}$ at that time, and statin therapy was started thereafter. Meanwhile, however, he did not continue to visit the hospital regularly; therefore, the statin therapy was discontinued 12 years prior to the patient's admission to our hospital in 2010.

In 2007, at 50 years of age, the patient was again diagnosed as having hypercholesterolemia and diabetes mellitus at a routine health check-up; however, medical treatment was not started at that time. In October 2009, he began to experience chest oppression on exertion. The chest pain was associated with cold sweating and subsided when the patient was at rest for five minutes. In May 2010, the duration of chest pain became prolonged up to 20 to 30 minutes and the frequency increased up to three to four times per day. The patient visited a regional hospital where electrocardiogram showed ST-T segment changes suggestive of the presence of unstable angina pectoris. He was immediately hospitalized and an intravenous infusion of heparin sodium and isosor- bide dinitrate was started, which markedly, but not completely, relieved his symptoms. He was then transferred to our hospital for further evaluation and treatment.

On admission, the patient's consciousness was clear, his body temperature was 36.8 degrees and his blood pressure was $108 / 72 \mathrm{mmHg}$. His heart sounds were normal and no abnormal heart murmurs were audible. No abnormal neurological findings were noted. Chest X-ray and electrocardiogram showed no significant abnormalities on admission. Multiple nodular subcutaneous tumors were observed on the dorsum of the bilateral hands, the bilateral wrists, the soles of the bilateral feet and the bilateral Achilles tendons (Fig. 2A, B). Severe thickening of the bilateral Achilles tendons (right side: $40 \mathrm{~mm}$, left side: $30 \mathrm{~mm}$ ) was confirmed on an X-ray examination (Fig. 2C, D). Echocardiography demonstrated that the aortic valve had three cusps, while valvular calcification was not significant.

The laboratory data obtained on admission are shown in the Table. At the time of admission, the patient began taking lipid- and glucose-lowering drugs, including rosuvastatin calcium $(5.0 \mathrm{mg} / \mathrm{d})$, metformin hydrochloride $(750 \mathrm{mg} / \mathrm{d})$, pioglitazone hydrochloride $(15 \mathrm{mg} / \mathrm{d})$ and glimepiride $(3.0$ $\mathrm{mg} / \mathrm{d})$. He is a former smoker with a Brinkman index of 390. The serum levels of sitosterol and campesterol measured by means of gas chromatography (SRL, Co., Ltd., Tokyo, Japan) were $1.2 \mu \mathrm{g} / \mathrm{mL}$ and $1.9 \mu \mathrm{g} / \mathrm{mL}$, respectively, neither of which were elevated.

Coronary angiography showed multivessel coronary artery stenosis with $99 \%$ stenosis in the right coronary artery 

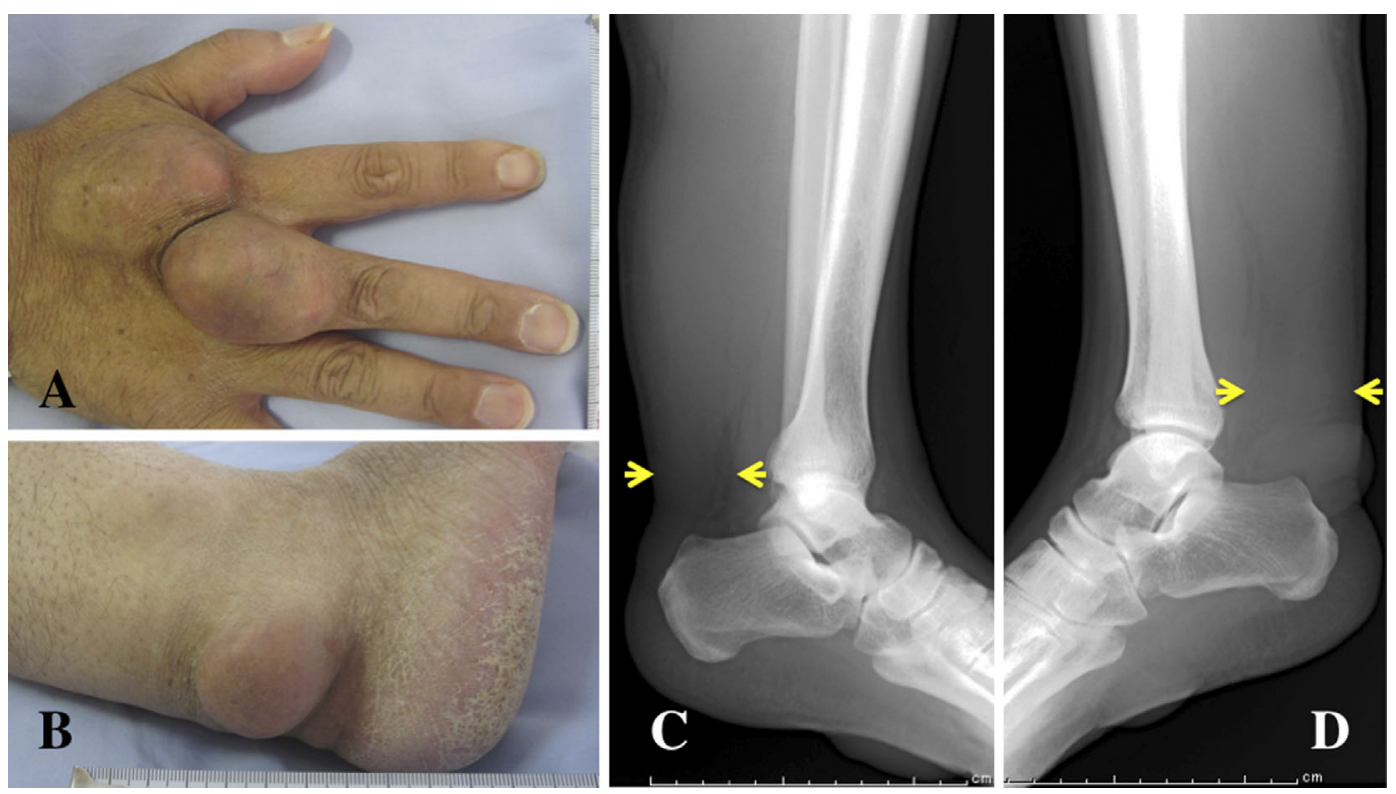

Figure 2. Large nodular tumors on the back side of the right hand (A) and the right Achilles tendon (B) are presented. Radiography of the Achilles tendons showed severe thickening of the bilateral Achilles tendons [left side (arrows in C): $30 \mathrm{~mm}$, right side (arrows in D): $40 \mathrm{~mm}$ ].

Table. Results of Laboratory Tests on Admission and 1 Month after the Treatment with a Statin and Anti-hyperglycemic Agents

\begin{tabular}{llll}
\hline & before & after & normal range \\
\hline Total Cholesterol & $282 \mathrm{mg} / \mathrm{dL}$ & $137 \mathrm{mg} / \mathrm{dL}$ & $(130-219)$ \\
LDL- Cholesterol & $208 \mathrm{mg} / \mathrm{dL}$ & $71 \mathrm{mg} / \mathrm{dL}$ & $(70-139)$ \\
HDL- Cholesterol & $45 \mathrm{mg} / \mathrm{dL}$ & $41 \mathrm{mg} / \mathrm{dL}$ & $(40-77)$ \\
Triglyceride & $143 \mathrm{mg} / \mathrm{dL}$ & $124 \mathrm{mg} / \mathrm{dL}$ & $(30-149)$ \\
Apolipoprotein B & $\mathrm{NE}$ & $79 \mathrm{mg} / \mathrm{dL}$ & $(73-109)$ \\
Lipoprotein (a) & $\mathrm{NE}$ & $23 \mathrm{mg} / \mathrm{dL}$ & $(<30)$ \\
Glucose & $232 \mathrm{mg} / \mathrm{dL}$ & $96 \mathrm{mg} / \mathrm{dL}$ & $(60-109)$ \\
HbA1c (JDS) & $9.3 \%$ & $6.2 \%$ & $(4.3-5.8)$ \\
\hline
\end{tabular}

NE: not examined, JDS: Japan Diabetes Society

(RCA), 90\% stenosis in the left anterior descending artery (LAD) and 99\% stenosis in the left circumflex artery (LCX) (Fig. 3A, B). No significant luminal stenosis was seen near the ostial lesions of the right or left coronary arteries. The motion of the left ventricle was within normal limits with an ejection fraction of $65.5 \%$. Coronary artery bypass graft surgery was performed on the 14th hospital day. The left internal thoracic artery was anastomosed to the LAD and saphenous vein grafts were anastomosed to the LCX and RCA, which relieved the patient's chest symptoms on exertion.

The results of the activity and gene analysis (BML Co., Ltd., Saitama, Japan) of the LDL receptor (LDLR) were as follows: the LDL-receptor activity in lymphocytes was $137 \%$ (normal rage: $>80 \%$ ) and none of the six common LDLR gene mutations of E119K, C317S, 1847T/C, L547V, P664L and K790X were detected.

In addition, DNA sequencing of all of the exons of LDLR and proprotein convertase subtilisin/kexin type 9 (PCSK9) did not show any gene mutations. On the other hand, sequencing of low-density lipoprotein receptor adaptor protein
1 (LDLRAP 1), which is also known as autosomal recessive hypercholesterolemia (ARH), revealed two mutations. A new DNA sequence variation in LDLRAP1 was found, as follows: $604 \mathrm{~T}>\mathrm{C}$ and $654 \mathrm{~A}>\mathrm{G}$. The frequency of $604 \mathrm{~T}>\mathrm{C}$ was $0.48 / 0.52$ and that of $654 \mathrm{~A}>\mathrm{G}$ was $0.50 / 0.50$ among 31 normal control subjects. The patient had the $\mathrm{C} / \mathrm{C}$ variation in 604 and the $\mathrm{G} / \mathrm{G}$ variation in 654 . They were both considered to be single nucleotide polymorphisms. We also sequenced the apolipoprotein B (APOB) gene. The G to A mutation at nucleotide 10,708 , the major cause of mutations in familial defective APOB, was not detected using asymmetric polymerase chain reaction (PCR) (3) in this patient. The nucleotides 10,564 to 10,884 on Exon 26 of the APOB gene were sequenced in this patient and found to be normal. The sequences of the primers used in this study for sequencing LDLRAP1 (4), LDLR, PCSK9 and APOB are described in the supplementary file.

\section{Discussion}

Currently, more than 1,100 mutations occurring at different sites on the LDLR gene in patients with FH have been reported (5). It has been reported that LDLR gene mutations can be identified in $67.8 \%$ of FH patients in the Japanese population (6). In the present case, on the other hand, we were unable to identify LDLR gene mutations by screening six commonly observed sites. Patients with these six mutations comprise approximately $30 \%$ of the total number of FH heterozygotes investigated in the Japanese population (6). Taking into consideration the finding that the LDLR activity in lymphocytes was within the normal range in this patient, a nonLDLR-mediated mechanism may underlie the pathophysiology in this patient, although the possibil- 


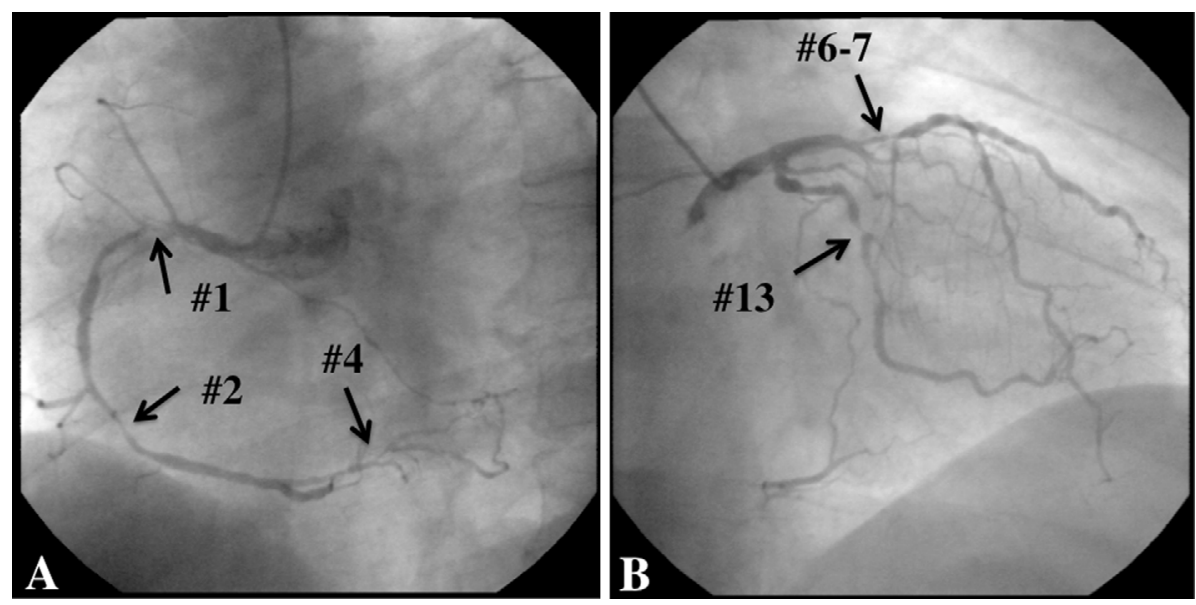

Figure 3. Coronary angiography revealed severe multivessel disease with $99 \%$ stenosis at segment (\#) $1,90 \%$ stenosis at \#2, 99\% stenosis at \#4 of the right coronary artery (A), 90\% stenosis at \#6-7 of the left anterior descending artery and $99 \%$ stenosis at \#13 of the left circumflex artery (B).

ity remains that a mutation of the LDLR gene other than these six mutations may have been present. However, on the other hand, compared with the LDLR gene mutation, fewer studies appear to have investigated the relationship between the LDLR genotype and its activity. A significant minority of patients (approximately 5\%) who fulfill the criteria for FH with angiographically-proven coronary disease do not have a defective LDLR function or a detectable mutation in the LDLR gene (7).

Recently, several other genes that are candidates that may explain the $\mathrm{FH}$ phenotype have been reported, including APOB, PCSK9 and ARH (8). These nonLDLR mutations are relatively rare compared with the LDLR gene mutations whose prevalence of homozygote and heterozygote in $\mathrm{FH}$ are estimated to be $1 / 1 \times 10^{6}$ and $1 / 500$, respectively. Additionally, the homozygote and heterozygote APOB are estimated to be $1 / 4 \times 10^{6}$ and $1 / 1,000$, respectively, the heterozygote of PCSK9 is estimated to be $<1 / 2,500$ and the heterozygote of $\mathrm{ARH}$ is estimated to be $<1 / 5 \times 10^{6}$. In addition to searching for common LDLR mutations, we performed sequencing of the exons of the LDLR gene, the PCSK9 gene, the ARH gene and the APOB gene; however, no mutations that could explain the overt dyslipidemia observed in our patients were observed.

Xanthomas are a characteristic feature of FH and most usually measure a few centimeters in diameter (9). Multiple large tendinous xanthomas and advanced coronary artery atherosclerosis were noted in the present case. It has been reported that the presence of xanthomas increases the risk of cardiovascular disease in FH patients by as much as three times, suggesting that xanthomas and atherosclerosis may share a certain etiology (10). It has also been suggested that the severity of atherosclerosis and tissue lipid deposition, including the development of xanthomas and the width of the Achilles tendon, is correlated with the duration of hypercholesterolemia or the cholesterol-year score (11). It is not well known whether there is a correlation between the severity of the phenotype of $\mathrm{FH}$, including multiple xanthomas and vas- cular diseases, and the genotype, including the sites of LDLR gene mutations. In our case, irrespective of the further extensive search for mutations in the LDLR, PCSK9, ARH and APOB genes, we were not able to identify any mutations in these genes that may potentially explain the patient's phenotype. Possible mechanisms, other than the genetic mutations examined in the current study, underlying prominent hypercholesterolemia, as observed in our patient, should be researched and identified in future studies.

The authors state that they have no Conflict of Interest (COI).

\section{Acknowledgement}

This study was partly supported by a research grant for the investigation of intractable diseases from the Ministry of Health, Labor and Welfare of Japan.

\section{References}

1. Marks D, Thorogood M, Neil HAW, Humphries SE. A review on the diagnosis, natural history, and treatment of familial hypercholesterolaemia. Atherosclerosis 168: 1-14, 2003.

2. van Aalst-Cohen ES, Jansen AC, Tanck MW, et al. Diagnosing familial hypercholesterolaemia: the relevance of genetic testing. Eur Heart J 27: 2240-2246, 2006.

3. Schuster H, Rauh G, Muller S, Keller C, Wolfram G, Zollner N. Allele-specific and asymmetric polymerase chain reaction amplification in combination: a one step polymerase chain reaction protocol for rapid diagnosis of familial defective apolipoprotein B-100. Analytical Biochemistry 204: 22-25, 1992.

4. Harada K, Miyamoto Y, Morisaki H, et al. A novel Thr56Met mutation of the autosomal recessive hypercholesterolemia gene associated with hypercholesterolemia. J Atheroscler Thromb 17: 131140, 2010.

5. Goldstein JL, Brown MS. History of discovery: the LDL receptor. Arterioscler Thromb Vasc Biol 29: 431-438, 2009.

6. Miyake Y, Yamamura T, Sakai N, Miyata T, Kokubo Y, Yamamoto A. Update of Japanese common LDLR gene mutations and their phenotypes. Mild type mutation L547V might predominate in the Japanese population. Atherosclerosis 203: 153-160, 2009.

7. Sun XM, Patel DD, Knight BL, Soutar AK; the Familial Hypercholesterolaemia Regression Study Group. Comparison of the ge- 
netic defect with LDL-receptor activity in cultured cells from patients with a clinical diagnosis of heterozygous familial hypercholesterolemia. Arterioscler Thromb Vasc Biol 17: 3092-3101, 1997.

8. Rader DJ, Cohen J, Hobbs HH. Monogenic hypercholesterolemia: new insights into pathogenesis and treatment. J Clin Invest 111: 1795-1803, 2003.

9. Weiss SW, Goldblum JR. Soft Tissue Tumors. 5th ed. Mosby Elsevier, Philadelphia, 2008: 355-358.

10. Oosterveer DM, Versmissen J, Yazdanpanah M, Hamza TH, Si- jbrands EJG. Differences in characteristics and risk of cardiovascular disease in familial hypercholesterolemia patients with and without tendon xanthomas: a systematic review and meta-analysis. Atherosclerosis 207: 311-317, 2009.

11. Schmidt HH, Hill S, Makariou EV, Feuerstein IM, Dugi KA, Hoeg JM. Relation of cholesterol-year score to severity of calcific atherosclerosis and tissue deposition in homozygous familial hypercholesterolemia. Am J Cardiol 77: 575-580, 1996.

(C) 2013 The Japanese Society of Internal Medicine http://www.naika.or.jp/imonline/index.html 